\title{
Research and Application on the Deceleration Sled Simplified Finite Element Simulation
}

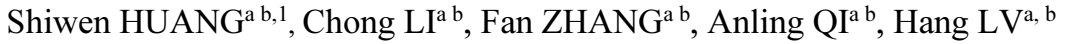 \\ ${ }^{a}$ CATARC Automotive Inspection Center (Wuhan) Co., Ltd., Wuhan, China \\ ${ }^{\mathrm{b}}$ China Automotive Technology and Research Center Co., Ltd., Tianjin, China
}

\begin{abstract}
Based on the basic structure of deceleration sled, the paper established an simplified finite element model by Ansys/Ls-Dyna software, which is verified by GB 13057-2014 passenger seat simulating impact test and GB 27887-2011 child seat simulating impact test. Then, how the sled mass and the layout of the steel bars influence the decelerating curves are being discussed through the FE calculation: when the layout of the steel bars and the initial velocity of the sled remain unchanged, the heavier the sled is, the longer the deceleration curve continued; when the sled mass and the initial velocity remain unchanged, the more centrally the steel bars are being placed, the larger the peak value of the deceleration curve is. What's more, the finite element model in this paper can provide some guidance in the daily tests.
\end{abstract}

Keywords. Deceleration sled, finite element simulation, simulation impact, deceleration curve

\section{Introduction}

Vehicle passive safety has been paid close attention to for a long time. According to statistics, from 2000 to 2019, as the development of traffic laws and regulations related to passive safety, road traffic death toll per 10 thousand vehicles has declined every year, which is shown in figure 1.

At the same time, as the rapid increase of national knowledge level, automobile safety has been paid greater attention to in automobile purchase. Thus, more and more auto makers have been concerned in vehicle passive safety research.

Passive safety test mainly covers vehicle impact test and simulated impact test, in which vehicle impact test usually costs a lot. However, during the early stage of vehicle research and development, lots of safety performance development and validation tests of auto parts are needed to be done. Thus, simulated impact tests are chosen in the early stage of passive safety development.

Deceleration sled system is very cheap and easy to use, which can conduct simulated impact tests repeatedly. Deceleration sled system can meet the requirements of Chinese standards, ECE regulations, Australia regulations and GSO regulations. The key components of deceleration sled system are a hydraulic pulldown, an energy absorber

\footnotetext{
${ }^{1}$ Corresponding Author, Shiwen HUANG, CATARC Automotive Inspection Center (Wuhan) Co., Ltd., Wuhan 430056, China; E-mail: huangshiwen@catarc.ac.cn.
} 
and a sled with a impulse lever, and the energy absorber mainly includes some steel bars of 6mm's diameter and some orientation bolts for fixing steel bars, as shown in figure 2 .

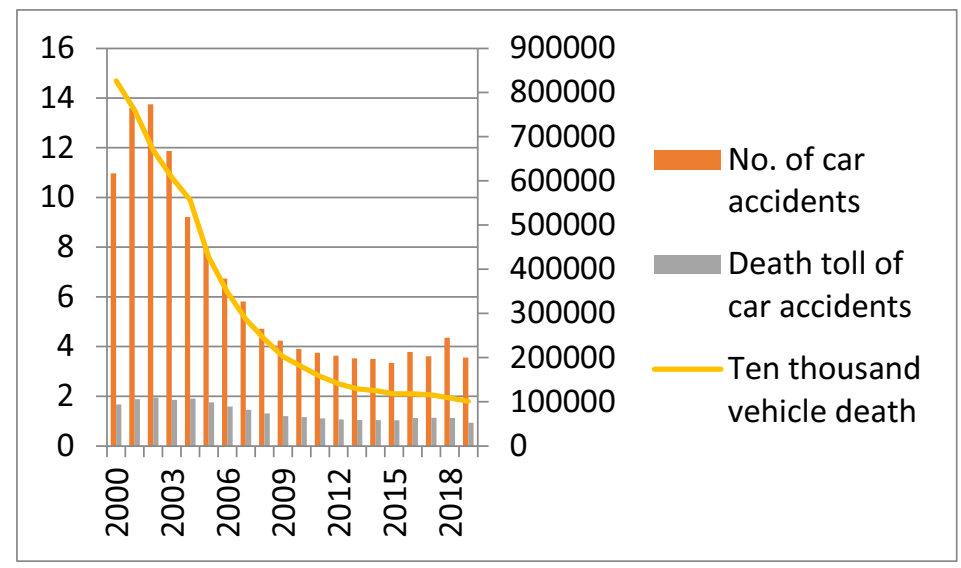

Figure 1. Cartogram for car accidents and death toll

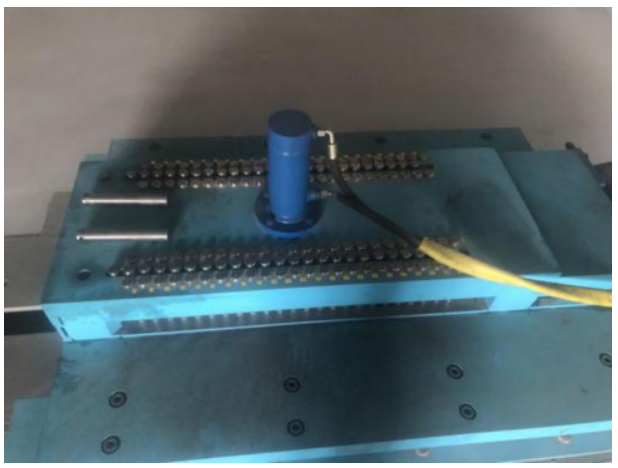

Figure 2. The energy absorber of deceleration sled system

The deceleration curves of simulated impact test are controlled by the steel bars in energy absorber of deceleration sled system, of which the working principles are explained as follows: according to the peak value and the duration of the peak, numbers, location and length of the steel bars will be adjusted. Firstly, steel bars are set out in the energy absorber in accordance with related regulations. Secondly, the energy absorber will start to pre-deform the steel bars in order to fix them and increase the roll damping effects of the steel bars. Thirdly, the sled including the impulse lever impact the energy absorber with a initial velocity, when the deceleration of the curve goes up, the peak value of the curve is decided by the number and length of steel bars. If the steel bars fall out during the impact the peak value of the curve will decline then. The moment when the peak occurs is influenced by the location and length of steel bars, which is shown as the slope of the curve. When steel bars are set out intensively, the slope of the curve will increase; while the sled hit against a vacancy or some short steel bars, which will be separated from the energy absorber during the impact, the slope of the curve will decrease.

Against the problems of the layout of steel bars in the deceleration sled, a lot of engineers and scholars have carried out many research. Xiaodong Chen[1] discussed the reproduction of the deceleration curves through theoretical calculation method. Shikuan 
Deng[2] expounded the principles of the deceleration sled, and explained the relationship between the deceleration sled and the slope of the deceleration curves according to the accumulative test data. Zhenyuan Tan[3] established and validated the reliability and availability of the finite element simulation model of the deceleration sled, improving the efficiency and accuracy of the sled. Changjiang Du[4] researched the layout of the steel bars through test method, in order to acquire some deceleration curves satisfied with relevant standards. Huiyun Zhang[5] studied the means to reproduce the deceleration curves, and summarized some layouts of steel bars by means of test method, largely reducing the debugs of deceleration curves, summing up some laws of deceleration curve reproduction. A large number of studies show that appropriate layouts of steel bars will stabilize the deceleration curves, satisfying various kinds of detection and tests, which follows that some factors such as the layouts, numbers and length of steel bars will decide whether the deceleration curves of simulation impact test satisfying relevant standards or not.

\section{Simplified Finite Element Model of the Deceleration Sled}

\subsection{Introduction of Simplified Fe Model of the Deceleration Sled}

According to some research and observations, during the simulation impact of the deceleration sled, the main parts participating in the impact are the energy absorber and the sled with a impulse lever, in which steel bars are fixed and pre-deformed through the energy absorber. Thus, in the energy absorber, except steel bars deforming during the impact and orientation bolts fixing steel bars, other parts can be regarded as rigid bodies which won't deform during the impact. Meanwhile, the sled with a impulse lever will hit against the energy absorber with a initial speed without deformation. Therefore, it can also be regarded as a rigid simplified impact body, the mass and initial speed of which are the same as the sled. In conclusion, in order to simplify the finite element model and reduce the calculation time, a simplified FE calculation model is designed via Ansys/Lsdyna software, shown as figure 3 . The model includes steel bars, orientation bolts and the rigid simplified ram, which can simulate the working procedure of the deceleration sled.

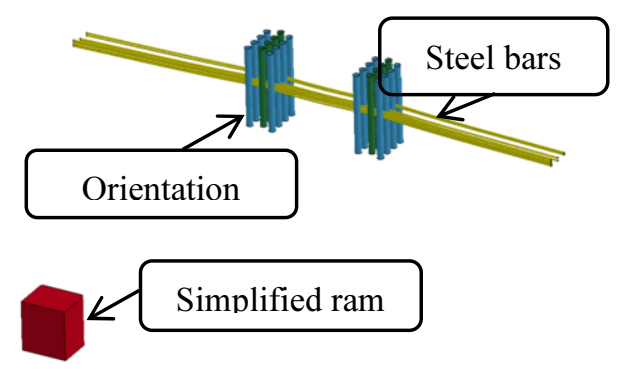

Figure 3. Simplified FE calculation model of the deceleration sled

In the FE model, the rigid simplified ram equipped with the mass of the total sled will simulate the sled and its impulse lever, in which as well as orientation bolts is set as 
rigid body, which means they will not participate in deformation during the impact. Firstly, the FE calculation model simulate the pre-deformation of the steel bolts loaded by the hydraulic device, shown as in figure 4(a). Secondly, the rigid simplified ram will be attached an initial velocity, crashing against the pre-deformed steel bars, where the steel bars will absorb kinetic energy and the deceleration curves are produced, as shown in figure $4(b)$.

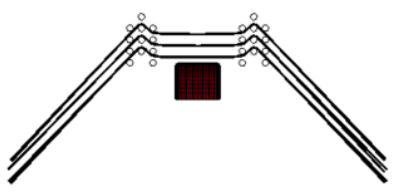

(a)

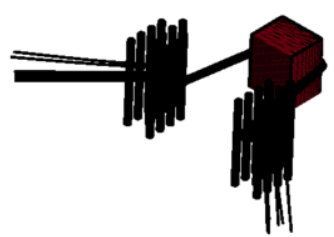

(b)

Figure 4. Deformation of steel bars (pre-deformation and deformation during the impact)

\subsection{Material Models}

In the working process of the deceleration sled, the orientation bolts and the rigid simplified ram will not deform. Hence, for simplifying the calculation model and reducing the calculation time explained above, the bolts and the ram will be set as rigid bodies, of which the material model is shown in table 1 . The material of steel bars is Q235 carbon steel, of which is the piecewise linear elastoplastic model, shown in table 2 , and the real stress-strain curve of the material is shown in figure 5, which is gained according to tensile tests.

Table 1. Material parameters of the rigid bodies

\begin{tabular}{ccc}
\hline Density $(\mathrm{kg} / \mathrm{m} 3)$ & Young's modulus $(\mathbf{P a})$ & Poisson's ratio \\
\hline 7850 & $2.1 \mathrm{e} 11$ & 0.3 \\
\hline
\end{tabular}

Table 2. Material parameters of steel bars

\begin{tabular}{cccc}
\hline Density $(\mathrm{kg} / \mathrm{m} 3)$ & Young's modulus $(\mathbf{P a})$ & Poisson's ratio & Density $(\mathrm{kg} / \mathbf{m 3})$ \\
\hline 7850 & $2.1 \mathrm{e} 11$ & 0.3 & $2.39 \mathrm{e} 8$ \\
\hline
\end{tabular}




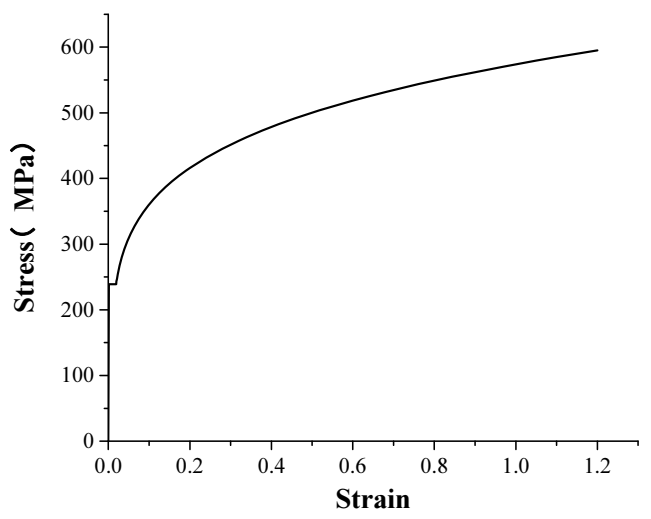

Figure 5. Real stress-strain curve of steel bars

\subsection{Validation of the Simplified Fe Model}

For validating the effectiveness of the simplified FE model of deceleration sled, this paper has selected the deceleration curve in "GB 13057-2014 Strength of the seats and their anchorages of passenger vehicles"[6] and the rear collision deceleration curve in "GB 27887-2011 Restraining devices for child occupants of power-driven vehicles"[7] to make comparisons between the real simulation impact tests and finite element calculations. The software for FE calculation is Ansys/Ls-dyna. On the basis of the requirements in the two standards, the initial velocity of deceleration curves of "GB 13057-2014" and "GB 27887-2011" is both set as $31 \mathrm{~km} / \mathrm{h}$, in which the energy exchanging curves of FE calculation are shown in figure 6 , while the deceleration curves are shown in figure 7.

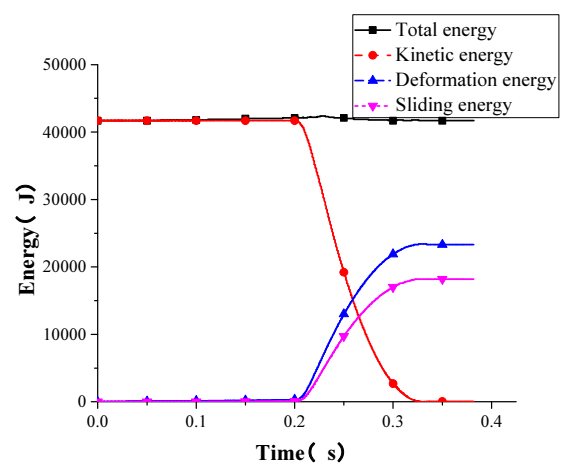

(a)

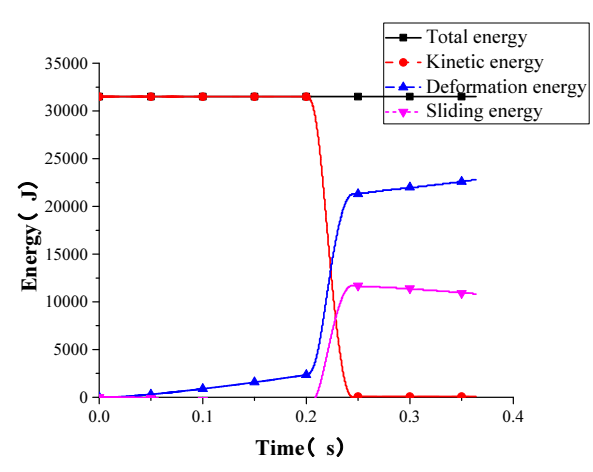

(b)

Figure 6. Energy exchange in the FE calculation (GB 13057-2014 and GB 27887-2011 standards)

As shown in figure 6, the whole energy in simulation impact system comprises the kinetic energy from rigid simplified ram, the deformation energy of steel bars and the sliding energy between steel bars and orientation bolts, in which the initial kinetic energy will transform into deformation energy and sliding energy gradually, which means that when the rigid ram gets contact with steel bars with an initial velocity, steel bars will begin to deform and slide with orientation bolts to produce energy until the velocity of the ram goes at 0 . 


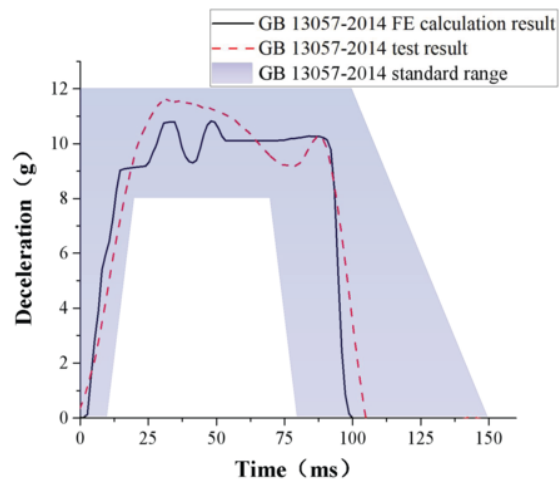

(a)

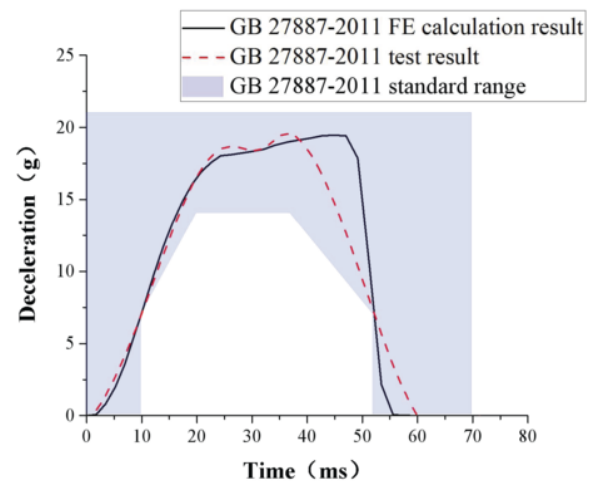

(b)

Figure 7. Comparisons of deceleration curves between FE calculations and tests ((a): GB 13057-2014 and (b): GB 27887-2011 standards)

It is observed that the deceleration curves from FE calculation and real test are both in the range of the standard range, as seen in figure 7. In figure 7(a), the slopes of the ascent stage, declining stage and the duration time in FE deceleration curves are almost the same as that in real test deceleration curves, and there is just a little difference in the peak values. In figure 7(b), the slope of the ascent stage, the deceleration peak value and the duration time in FE deceleration curves are almost the same as that in real test curves, and there is just a little difference in the slope of the declining stage. In general, the timedeceleration curves calculated by FE model are very close to the curves gained by real tests. Thus, the simplified FE calculation model has been validated.

\section{Influence in Deceleration Curves from the Layout of Steel Bars and the Mass of the Sled}

In the real tests during laboratory work, if a new standard is met, or the client provides a new deceleration curve, it will cost a lot of time in the calibration of deceleration curves when using the deceleration sled. In order to reduce the time and property cost in the test calibration, the validated simplified FE calculation model will be used in this chapter. Moreover, the relationship between the mass of the sled and the deceleration curves, as well as the relationship between the layout of steel bars and the deceleration curves will be discussed based on "GB 13057-2014" standard, where the reasonability of using simplified FE model in estimating deceleration curve will be investigated. 


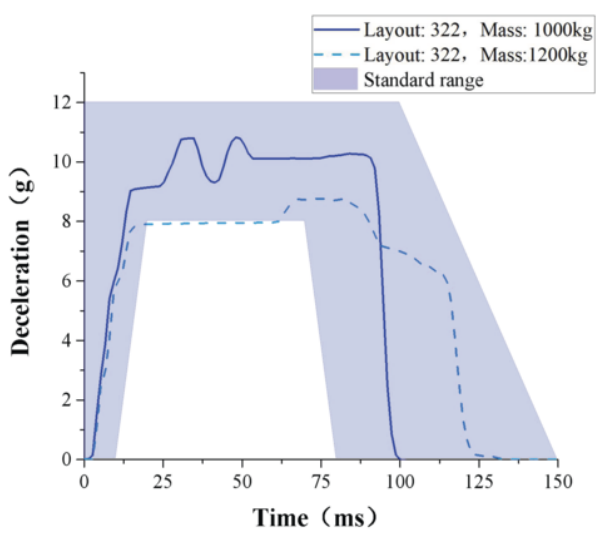

(a)

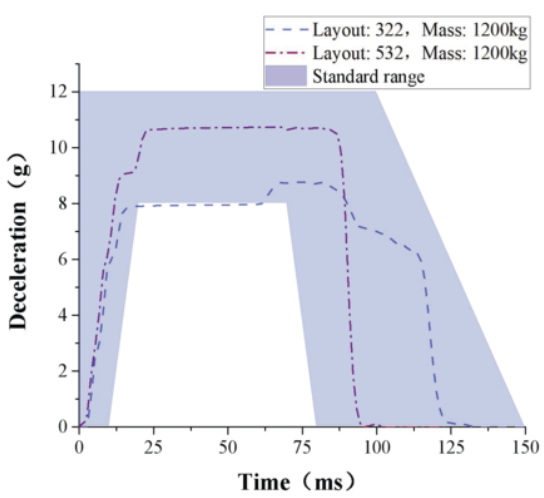

(b)

Figure 8. The relationship between the layout of steel bars, the mass of the sled and deceleration curves ((a): the relationship between the mass of the sled and deceleration curves; (b): The relationship between the layout of steel bars and deceleration curves)

In figure 8, "Layout: 322" means that in the energy absorber of the deceleration sled, there are relatively 3, 2, 2 steel bars in the first, second, third row in the orientation bolts alignment, which is shown in the FE model in figure 4(a). Meanwhile, "Layout: 532" means that there are relatively 5, 3, 2 steel bars in the first, second, third row in the orientation bolts alignment.

In figure 8(a), the curve of dark blue represents that the corresponding deceleration sled mass is $1000 \mathrm{~kg}$, and the layout of steel bars is " 322 ", and the curve of light blue means that the corresponding deceleration sled mass is $1200 \mathrm{~kg}$, and the layout of steel bars is " 322 ".

According to the law of conservation energy, when the number and layout of steel bars, and the initial speed of the deceleration sled remain unchanged, the larger the mass of the deceleration sled is, the more energy will be needed to decelerate the sled in the test. However, as shown in figure 8(a), the peak value of the deceleration curve of the light blue (the corresponding sled mass is $1200 \mathrm{~kg}$ ) is less than that of the dark blue (the corresponding sled mass is $1000 \mathrm{~kg}$ ), which means that "Layout 322 " cannot provide enough deformation energy to produce large enough deceleration. In consequence, it is needed to change the layout of steel bars, and adopt "Layout 532" in the FE calculation. As shown in figure 8(b), when the steel bars become more, the peak value of the curve will become larger, and the duration time of the curve will be less. It follows that during the test, increasing the mass of the sled can extend the duration time of the curve, which means fixing some mass block on the sled; while increasing the number of the steel bars at front rows can increase the peak value of the curve and shorten the duration time.

\section{Conclusions}

The paper firstly established a simplified finite element simulation model via Ansys/Lsdyna software, and validated the FE calculation model according to real tests. Secondly, the relationship between the mass of the sled and the deceleration curve as well as the relationship between the layout of steel bars and the deceleration curve are discussed using the validated FE calculation model: when the layout of steel bars and the initial 
velocity of the sled remain unchanged, the heavier the sled is, the longer the duration time of the deceleration curve will be; when the mass and the initial velocity of the sled remain unchanged, the more intensively steel bars are layout, the larger the peak value of the deceleration curve will be. Therefore, if longer duration time is needed, the way of adding up the mass of the sled may be adopted; if larger peak value and less duration time are needed, the way of increasing the number of steel bars at front rows of the energy absorber may be adopted. Meanwhile, the simplified FE calculation model can provide some guidance for the new deceleration curves that some clients needed.

\section{References}

[1] Xiaodong Cheng, Siyi Shang, Ruhai Ge, et al. A Research on an Energy Absorber for Vehicle Sled Test of Crash Simulation [J]. AUTOMOTIVE ENGINEERING, 2006, 028(007):659-662.

[2] Shikuan Deng, Chengye Ning, Fuzhen Hou, et al. The Research on the energy absorption device of the deceleration sled [C]//2012 the 15th Automobile Safety Technology Academic Conference of Chinese Society of Automotive Engineering. 2012.

[3] Zhenyuan Tan, Xin Wang, Changzheng Yan, et al. Simulation Research and Test Application of the Sled Impact Test Waveform Repetition [C]//2012 China Academic Journal Electronic Publishing House. 2012.

[4] Changjiang Du, Chunwang Yang, Biao Zhang, et al. Experimental Debug and Research of Energy Absorber for Dynamic Sled [J]. Automobile Science \& Technology, 2014(4).

[5] Huiyun Zhang, Lizhi Liu, Bin Yang, et al. Application and Curve Reproduction of the Deceleration Sled Waveform Generator [J]. Automobile Science \& Technology, 2015(1):57-62.

[6] GB 13057-2014 Strength of the seats and their anchorages of passenger vehicles.

[7] GB 27887-2011 Restraining devices for child occupants of power-driven vehicles. 